Rapid Reviews COVID-19

\title{
Review 2: "Vaccination for some childhood diseases may impact the outcome of covid-19 infections"
}

\section{Jagdish Shukla ${ }^{1}$}

${ }^{1}$ University of Montana

Published on: Jan 28, 2022

License: Creative Commons Attribution 4.0 International License (CC-BY 4.0). 


\section{$\underline{\text { RR:C19 Evidence Scale rating by reviewer: }}$}

- Potentially informative. The main claims made are not strongly justified by the methods and data, but may yield some insight. The results and conclusions of the study may resemble those from the hypothetical ideal study, but there is substantial room for doubt. Decision-makers should consider this evidence only with a thorough understanding of its weaknesses, alongside other evidence and theory. Decisionmakers should not consider this actionable, unless the weaknesses are clearly understood and there is other theory and evidence to further support it.

$* * * * * * * * * * * * * * * * * * * * * * * * * * * * * * * * * * * * * * *$

\section{Review:}

It is a very debatable topic and needs more ongoing research. This article was written based on information from August 2020 and data around that time. Vaccinations against COVID-19 were not available at that time. There are several studies that show that certain vaccination, like BCG, may provide some potential cross-immunity to several other viral infections. Their results showed statistically significant differences between the mean COVID-19 death rate between vaccinated and unvaccinated countries for BCG, IPV, and HB-BD. The authors have acknowledged several limitations to the study, and there are several confounding factors. I recommend collecting more data on \% death rate and recovery rate for high, middle, and low-income countries for BCG, IPV, and HB-BD. I recommend more studies on a larger scale to determine further impact. 